\title{
Expression and interpretation of relative risk and odds ratio in biomedical research studies
}

\section{Suresh K Sharma ${ }^{1}$, Shiv Kumar Mudgal ${ }^{2}$, Jitender Chaturvedi ${ }^{3}$}

${ }^{1}$ Professor \& Principal, College of Nursing; All India Institute of Medical Sciences, Rishikesh-249203 Uttarakhand; ${ }^{2}$ Tutor, College of Nursing; All India Institute of Medical Sciences, Rishikesh-249203 Uttarakhand; ${ }^{3}$ Assistant Professor, Department of Neurosurgery, All India Institute of Medical Sciences, Rishikesh-249203 Uttarakhand, India

\begin{tabular}{|c|c|c|c|c|c|c|c|c|}
\hline Abstract & Introduction & Methodology & Results & Conclusion & References & Citation & Tables / Figures & \\
\hline \multicolumn{9}{|c|}{ Corresponding Author } \\
\hline \multicolumn{8}{|c|}{$\begin{array}{l}\text { Prof Suresh K Sharma, College of Nursing, All India Institute Of Medical Science, Rishikesh-249203 } \\
\text { Uttarakhand, India } \\
\text { E Mail ID: } \underline{\text { skaiims17@gmail.com }}\end{array}$} & 口ing \\
\hline
\end{tabular}

\section{Citation}

Sharma SK, Mudgal SK, Chaturvedi J. Expression and interpretation of relative risk and odds ratio in biomedical research studies. Indian J Comm Health. 2020;32(2):344-347.

Source of Funding: Nil Conflict of Interest: Nil

\section{Article Cycle}

Received: 30/03/2020; Revision: 15/04/2020; Accepted: 25/05/2020; Published:30/06/2020

This work is licensed under a Creative Commons Attribution 4.0 International License.

\section{Abstract}

Relative risk and odds ratio are commonly used in the biomedical research studies; however, expression and interpretation must be done very carefully. A risk ratio and an odds ratio are used in cohort studies but only odds ratio is used in case control studies. However, relative risk or risk ratio is found to be frequently used in the interventional biomedical research studies. The relative risk and odds ratio provide important information regarding the effect of a risk factor on the outcome of interest. The relative risk and odds ratio of 1 suggests that there is no difference between two groups. A value $>1$ suggests increase risk, while a value $<1$ suggest reduction of risk. If the confidence interval meets or includes value 1.00 (line of no difference) indicates there is no difference between the groups.

\section{Keywords}

Biomedical research; odds ratio; risks; relative risk

\section{Introduction}

Many research articles explain their results in terms of relative risk (RR) and odds ratio (OR) to interpret the whether results are significant or not for categorical outcomes such as results of a meta-analysis reported effect of heparin and normal saline to maintain patency of central venous catheter and presented by risk ratio 0.83 , $95 \% \mathrm{Cl} 0.50-1.40 ; P=0.13$.(1) While, these two widely used terms have been discussed in various articles for a novice researcher it is still difficult to interpret the odds ratio and relative risk. Therefore, in this article authors tried to discuss interpretation of these terms in simple and practical language with examples. Reader may note that the examples given in this article are hypothetical.

\section{Relative Risk (RR)}

The relative risk (RR) is probability of an incident occurrence after exposure to a risk in one group compared with the probability of its occurrence in placebo/other group.(2)

\section{Worked Example:}

Let us start with an assumed example that a researcher conducted a cohort study to assess the risk of infections when emergency craniotomies were performed on patients with intracranial hematoma after road traffic accidents. Researcher presented the data and calculated the relative risk as follow:

Presentation of the data in a $2 \times 2$ contingency (Table 1 ) helps the readers in analyzing and interpretation of results. In table 1 it is clear that emergency craniotomy was undertaken in a total of 139 patients and eleven out of them got infections, then calculation of cumulative incidence by 11 divided by 139 , or $7.91 \%$. In the same way the cumulative incidence calculated in the patient for those who had not undergone an emergency craniotomy were 2 divided by 98 or $2.04 \%$. Therefore, the risk ratio is 7.91/2.04 or 3.8.

When we are interpreting the results of the above study, we say that individuals who had undergone emergency craniotomy had 3.8 times the risk of infection compared to those who did not undergo emergency craniotomy. (2) 
INDIAN JOURNAL OF COMMUNITY HEALTH / VOL 32 / ISSUE NO 02 / APR - JUN 2020 When Risk Ratio <1

If in a study, we find that the risk ratio is less than 1 ; this would recommend that there are less chances of risk in the exposure group. Let us understand this with an assumed example of results of a randomized control trial which was carried out to identify whether clipping was beneficial in decreasing mortality among patient with ruptured cerebral aneurysm. Information about this study is compiled in the $2 \times 2$ contingency (Table 2).

The appropriate interpretation of this value would be: Patients who underwent craniotomy and clipping had 0.38 times the risk of mortality compared to patients who were treated with coiling. Here it is important to remember that the comparison (placebo/other) group must be defined clearly. For example, if you directly write, "Patients who underwent craniotomy and clipping have 0.38 times the risk of mortality." then readers may have a question "Compared to what?" is it group of patients who did not undergo any craniotomy and clipping, those who underwent any medical management, those who underwent clipping along with any other intervention or those who underwent coiling and so on. $(2,3)$

In general, we can say that if:

- The risk ratio is 1 or near to 1 , it recommends that there is no difference or little difference in risk. It means the incidences in both groups are equal.

- The risk ratio $>1$ recommends a high risk of particular outcome of interest in the exposed group.

- The risk ratio $<1$ recommends a risk reduction in the exposed group.

\section{Percent Relative Effect}

We may look at and interpret these finding by computing the percent relative effect. In this we assume bassline risk in unexposed or control group as $100 \%$ and then interpret percent of risk in exposed group comparing with assumed bassline risk in unexposed or control group as $100 \%$. For example:

\section{- If Risk Ratio >1}

In post-operative infection research study, the group that had emergency craniotomy had a $280 \%$ high risk than the risk in the comparison group. As we consider that unexposed group has $100 \%$ risk.

percent $(\%)$ increase risk $=($ risk ratio -1$) \times 100$

Therefore, for post-operative infection study the percent $(\%)$ increase risk would be:

$(3.8-1) \times 100=280 \%$ risk increases

Interpretation: Patients who underwent emergency craniotomy had a $280 \%$ increase in risk of developing infection compared to those who did not undergo emergency craniotomy.

- When Risk Ratio < 1

For the clipping RCT, the patient who underwent craniotomy and clipping had a $62 \%$ less risk of mortality.

$\%$ decrease risk $=(1-$ Risk Ratio $) \times 100$
[Expression and interpretation...] | Sharma SK et al $(1-0.38) \times 100=62 \%$ risk reduction

Interpretation: Patients who underwent clipping had a $62 \%$ decreased risk of mortality compared to patients who underwent coiling.

It is important to understand that it is not correct to express or interpret that patient who had an emergency craniotomy had 3.8 times more risk or 3.8 times greater risk. Actually, patients with emergency craniotomy had a $280 \%$ increase in risk. Similarly, in the clipping RCT, it is wrong to express or interpret that patients who underwent clipping surgery had 0.38 times less risk. Actually, patients had 62\% less risk.(4)

\section{How to Interpret Correctly}

- Individuals who underwent emergency craniotomy had 3.8 times the risk of infection compared to individuals who did not undergo emergency craniotomy.

- Individuals who underwent emergency craniotomy were 3.8 times as likely to get infection compared to individuals who did not undergo emergency craniotomy.

- The risk of infection to individuals who underwent emergency craniotomy was 3.8 times as high as the risk of infection compared to individuals who did not undergo emergency craniotomy.

- $\quad$ Patients who underwent clipping had 0.38 times the risk of mortality compared to individuals underwent coiling.

- The risk of mortality among individuals who underwent clipping was 0.38 times as high as the risk of mortality among individuals who underwent coiling.

- $\quad$ Patients who underwent clipping had $62 \%$ less risk of mortality compared to individuals who underwent coiling.

Risk Ratio for three or more Groups with the application of Reference Group

We usually encounter some of the cohort studies or clinical trials which compare the risk of outcomes /disease occurrence among three or more than three groups. In this condition, information can be compiled in a table with three or more rows to have these groups. $(3,4)$

Let us understand it with an assumed example from a study. Suppose a study assessing the association between exposure to surgical treatment and risk of complications. In this study there are no unexposed participants and we allocated them in coiling, clipping and flow-diversion stenting approach. In this situation, it is judicious to have a reference group which is least exposed and compare other exposed groups against this.

(Table 3 ) represented that the group of individual who underwent flow-diversion stenting approach had the lowest cumulative incidence ( 0.0425 or 42.5 per 1000 over the period of observation), while clipping and coiling approach had medium ( 0.0588 or 58.8 per 1000 over the 
period of observation) and high ( 0.0816 or 81.6 per 1000

over the period of observation) cumulative incidences, respectively.

- $\quad$ Therefore, the risk ratio for medium exposed group compared to least exposed group (this is reference group) is $0.0588 / 0.0425=1.38$ and the RR for high exposed group compared to least exposed group (the reference) is $0.0816 / 0.0425=1.92$

Interpretation: Compared to individuals who underwent flow-diversion stenting approach, those who underwent clipping had 1.38 times the risk of re-bleeding (38\% risk increases), and those who underwent coiling had 1.92 times the risk ( $92 \%$ risk increases).

As the relative risk is a simple ratio, errors usually happen when we use words like 'more' or 'less'. So, interpret it by stating: 'persons who had (intervention group/name of exposure) relative risk "times the risk" compared to persons who (control group/group without exposure).' OR "The risk of (name of disease) among those who (name of exposure) was relative risk 'times as low/high as' the risk of (name of disease name) among persons who did not (exposure name).(4)

\section{Odds Ratio}

The Odds ratio is a ratio of the odds of an incident in an experimental group after exposure to a particular risk compared to the odds of same incident in a placebo/other/control group.(2)

\section{Worked Example:}

To understand about the expression and interpretation of an odds ratio we assumed the information presented in a true case control study about the rare disease in a given population (refer to Tabel-4). This presented in (Table 4):

$$
\begin{gathered}
\text { Odds Ratio }(\mathrm{OR})=\mathrm{ad} / \mathrm{bc} \\
=52 \times 290 / 28 \times 187 \\
=2.88
\end{gathered}
$$

- According to Table 4, we can say that individuals with carcinoma are 2.88 times more likely to be exposed to radiation than those without carcinoma.

- Another way to express: the odds of developing carcinoma with radiation exposure (interventional to placebo/control) are 2.88 to 1 .

- Here it is important to understand that it is not right way to interpret it as "individuals with radiation exposure are 2.88 times more likely to develop carcinoma than those without exposure." The reason is that a case control study begins from outcome i.e. selecting a sample with the outcome of interest which is carcinoma in this case.

- The interpretation of Odds ratio is same as relative risk. The value of 1.00 in odds ratio indicates that there is no difference in risk.

- When the value of OR is $<1.00$ it indicates a risk reduction of an incident/outcome of interest in the group that was exposed to the risk.
[Expression and interpretation...] | Sharma SK et al

- When the value of the OR is $>1.00$ it indicates an increased risk of an incident/outcome of interest in the exposed group.

- To determine that an OR value is statistically significant we must see the OR value, its $95 \%$ confidence interval $(\mathrm{Cl})$ and $\mathrm{p}$-value. If the confidence interval meets or cross value 1.00 (line of no difference) then we can interpret that $O R$ is not statistically significant.(5)

For example: The results section of an assumed study showed "The patients with infection at 72 hours were ... (adjusted odds ratio with routine surgery, $0.88 ; 95 \% \mathrm{Cl}$, 0.78 to $0.92 ; \mathrm{P}=0.005$ )

The odds of infection, cranial surgery at 72 hours after randomization in the routine surgery were $12 \%$ less than in the emergency surgery with the true population effect between $22 \%$ and $8 \%$. As the odds ratio did not cross the confidence interval 1 and $p$ value is less than 0.05 so we can interpret that the result was statistically significant.(6)

\section{Point to be remembered}

- The expression of odds ratio is difficult in plain English/languages and therefore, it is difficult to interpret and understand when compared to relative risk.

- When disease is very rare then OR and RR values are usually same or very close to each other but interpretation is different. For example, when we get same value 0.70 for OR and RR. Then RR can be expressed that intervention decreased the risk by $70 \%$ or intervention group had 0.70 times the risk of an incident compared to other/reference group. But in odds ratio researcher must expressed that for every 0.30 (or 30 ) people who experienced an incident in the interventional group, 1 people (or 100 people) will experience the incident in the placebo group. That is, the odds are 30 to 100 .

- We can use a risk ratio or an odds ratio in cohort studies but in case control studies we can compute one and only, the odds ratio.

\section{Conclusion}

The relative risk and odds ratio provide important information regarding the effect of a risk factor on the outcome of interest. The relative risk and odds ratio of 1 suggests that there is no difference between two groups. $A$ value $>1$ suggests increase risk while a value $<1$ suggest reduction of risk. If the confidence interval meets or includes value 1.00 (line of no difference) indicates there is no difference between the groups.

\section{References}

1. Sharma SK, Mudgal SK, Gaur R, Sharma R, Sharma M, Thakur K. Heparin flush vs. normal saline flush to maintain the patency of central venous catheter among adult patients: A systematic review and meta-analysis. J Family Med Prim Care. 2019 Sep;8(9):2779-2792. doi: 10.4103/jfmpc.jfmpc_669_19. 
eCollection 2019 Sep. Review. PubMed PMID: 31681643; PubMed Central PMCID: PMC6820433. [PubMed].

2. Andrade C. Understanding relative risk, odds ratio, and related terms: as simple as it can get. J Clin Psychiatry. 2015 Jul;76(7):e857-61. doi: 10.4088/JCP.15f10150. PubMed PMID: 26231012. [PubMed].

3. Bodemer N, Meder B, Gigerenzer G. Communicating Relative Risk Changes with Baseline Risk: Presentation Format and Numeracy Matter. Med Decis Making. 2014 Jul;34(5):615-26. doi: 10.1177/0272989X14526305. Epub 2014 May 6. PubMed PMID: 24803429. [PubMed].
[Expression and interpretation...] | Sharma SK et al

4. Simon SD. Understanding the odds ratio and the relative risk. J Androl. 2001 Jul-Aug;22(4):533-6. Review. PubMed PMID: 11451349.[PubMed].

5. Szumilas M. Explaining odds ratios. J Can Acad Child Adolesc Psychiatry. 2010 Aug;19(3):227-9. Erratum in: J Can Acad Child Adolesc Psychiatry. 2015 Winter;24(1):58.

6. What is an Odds Ratio and How do I Interpret It? - Critical Appraisal [Internet]. [cited 2020 Mar 2]. Available from: https://psychscenehub.com/psychpedia/oddss-ratio-2/

\section{Tables}

TABLE 1 Incidence of infection in patients undergoing emergency craniotomy vs. Other group

\begin{tabular}{|l|l|l|l|l|}
\hline Emergency craniotomy performed & Infection present & Infection absent & Total & Cumulative incidence \\
\hline Yes & 11 & 128 & 139 & $11 / 139=7.91 \%$ \\
\hline No & 2 & 96 & 98 & $2 / 98=2.04 \%$ \\
\hline
\end{tabular}

TABLE 2 Incidence of mortality in patients who had clipping vs. Coiling for management of ruptured cerebral aneurysm

\begin{tabular}{|l|l|l|l|l|}
\hline Intervention & Mortality & No mortality & Total & Cumulative Incidence \\
\hline Clipping & 7 & 610 & 617 & $7 / 617=0.0113$ \\
\hline Coiling & 18 & 595 & 613 & $18 / 613=0.0293$ \\
\hline
\end{tabular}

TABLE 3 Incidence of re-bleeding in patients undergoing clipping, coiling and flow-diversion stenting for management of ruptured cerebral aneurysm

\begin{tabular}{|c|c|c|c|c|}
\hline $\begin{array}{l}\text { Surgical approach/ } \\
\text { exposure }\end{array}$ & Re-bleeding & No re-bleeding & Total & Cumulative Incidence \\
\hline Clipping & 5 & 80 & 85 & $5 / 65=0.0588$ \\
\hline Coiling & 8 & 90 & 98 & $8 / 98=0.0816$ \\
\hline Stenting & 4 & 90 & 94 & $4 / 94=0.0425$ \\
\hline
\end{tabular}

TABLE 4 Comparing history of radiation exposure in patients with diagnosed carcinoma vs. Healthy controls

\begin{tabular}{|l|l|l|}
\hline Radiation Exposure & Carcinoma present & No Carcinoma \\
\hline Yes & 52 (a) & 187 (b) \\
\hline No & 28 (c) & 290 (d) \\
\hline
\end{tabular}

